\title{
ÜBERTRAGUNGSTECHNIK
}

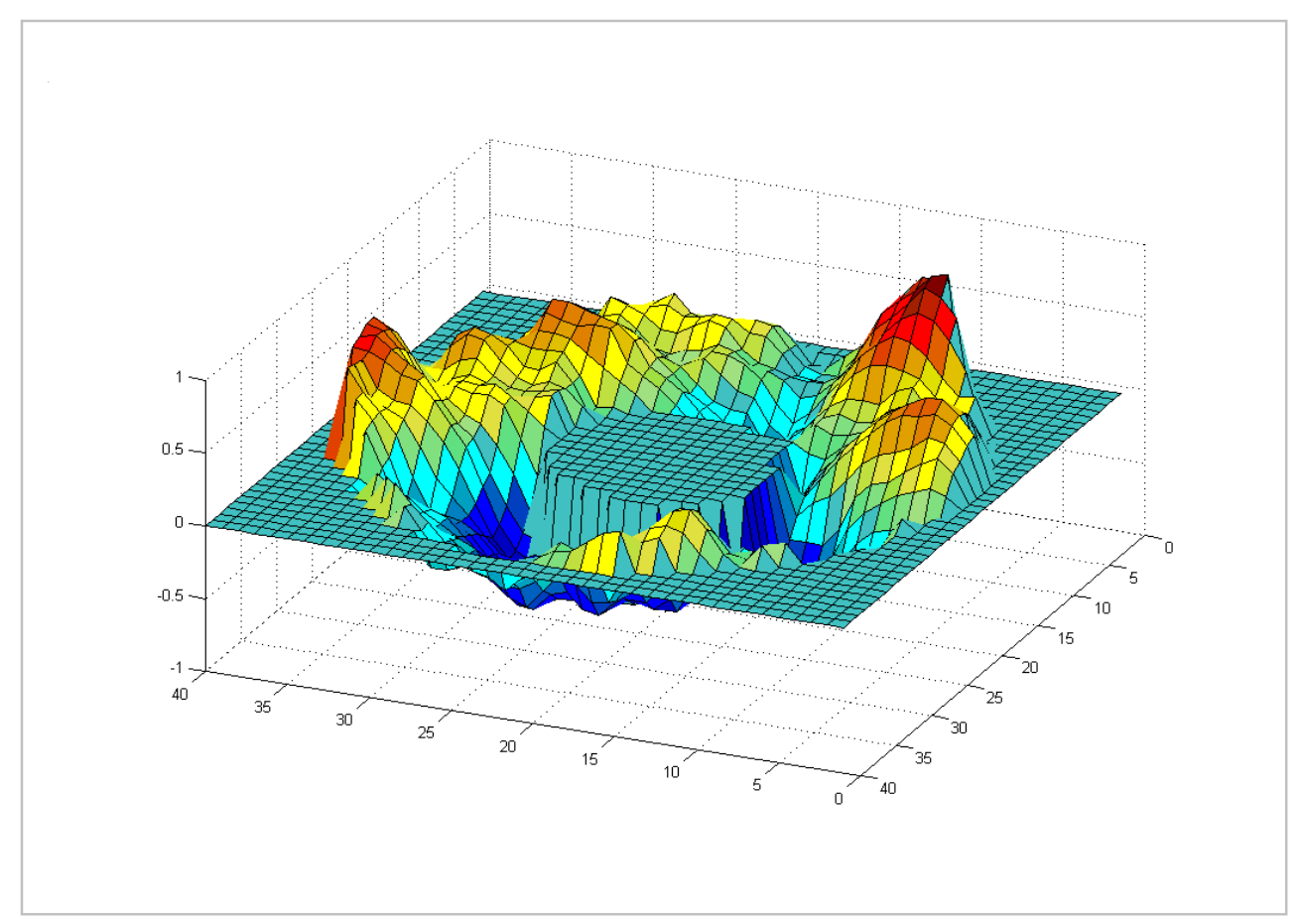

\section{Wavefront Measurements at ESA's Optical Ground Station and Simulation of Heterodyne Receiver Performance}

Dirk Giggenbach

Conference on Free Space Laser Communication Technology XII

24 January 2000, San José, USA

Proceedings of SPIE Vol. 3932 


\title{
Wavefront Measurements at ESA's Optical Ground Station and Simulation of Heterodyne Receiver Performance
}

\author{
Dirk Giggenbach \\ German Aerospace Center (DLR), D-82234 Wessling, Germany
}

\begin{abstract}
Future applications for the Optical Ground Station (OGS) on Tenerife, Spain, are currently being investigated, e.g. the use as a ground terminal in deep space missions. The impact of atmospheric effects on the sensitivity and reliability of an optical link depends heavily on the wavelength and the kind of transmission, mainly if direct or coherent detection is employed. To simulate and evaluate the link quality in the case of coherent reception (heterodyne or homodyne), measurements of the wavefront and intensity distributions have been carried out at the OGS telescope with a ShackHartmann sensor, using bright stars as reference sources. A representative set of normalized measurement samples has been generated and evaluated in respect of basic parameters like scintillations, tip-tilt, and wavefront error. The complex speckle patterns at the focal plane have been computed and a superposition with a local oscillator has been simulated. The outcome of these simulations (i.e. heterodyning efficiency) depending on several parameters is presented and compared with environmental conditions.
\end{abstract}

Keywords: optical ground station, wavefront measurements, optical free space links, coherent optical communications, atmospheric turbulence, heterodyne efficiency, SILEX

\section{INTRODUCTION}

The Optical Ground Station (OGS) on Tenerife, Spain, shall be used as a counter terminal in ESA's optical space communications experiment "SILEX" (Semiconductor Intersatellite Link Experiment) together with the experimental geostationary satellite ARTEMIS. Moreover, DLR and ESA are already evaluating future applications of the OGS infrastructure. Therefore a study has been carried out by Carl Zeiss Oberkochen together with the DLR Institute for Communication and Navigation evaluating the potential modification of the OGS towards coherent communications at $1.064 \mu \mathrm{m}$, e.g. with GEO relais satellites or with deep-space probes [OGS99]. The measurements underlying this paper have been done in the scope of this study.

It is obvious that atmospheric turbulence degrades the optical wavefront, which is a drawback especially for heterodyning receivers. But by more sophisticated techniques like multi-aperture or adaptive optics these effects can be reduced to an acceptable level. The advantage of coherent over direct detection free-space communications is especially strong with day-time operation due to the background light insensitivity. The aim of the measurements and their statistical analysis was to provide an estimate for the performance of an uncorrected coherent optical downlink to the OGS.

Published in "Conference on Free Space Laser Communication Technology XII", 24 Jan. 2000, SPIE Vol. 3932

Correspondence to: Dirk Giggenbach, Institute for Communication and Navigation, DLR-Oberpfaffenhofen, D-82234 Wessling Email: dirk.giggenbach@dlr.de

http://www.dlr.de/nt/nt-ue/members/digi 


\section{DESCRIPTION OF ESA'S OPTICAL GROUND STATION}

The telescope's 1m-aperture together with the central obscuration by the secondary mirror provides an effective reception area of $0.70 \mathrm{~m}^{2}$.

The telescope can be configured to produce either a focal spot at the Cassegrain focus (right behind the primary mirror) or one at the Coudéfocus. Therefore the beam is lead by mirrors through the mechanical axle and an evacuated tube down one storey into the Coudé-room, where the optical signal can be treated conveniently on an optical bench. For communications experiments only the Coudéconfiguration is used. The effective focal length in Coudé-configuration is $39 \mathrm{~m}$. The unvignetted field of view is $2.3 \mathrm{mrad}$ and the convergence angle into the focal spot is $28 \mathrm{mrad}$ [SOD].

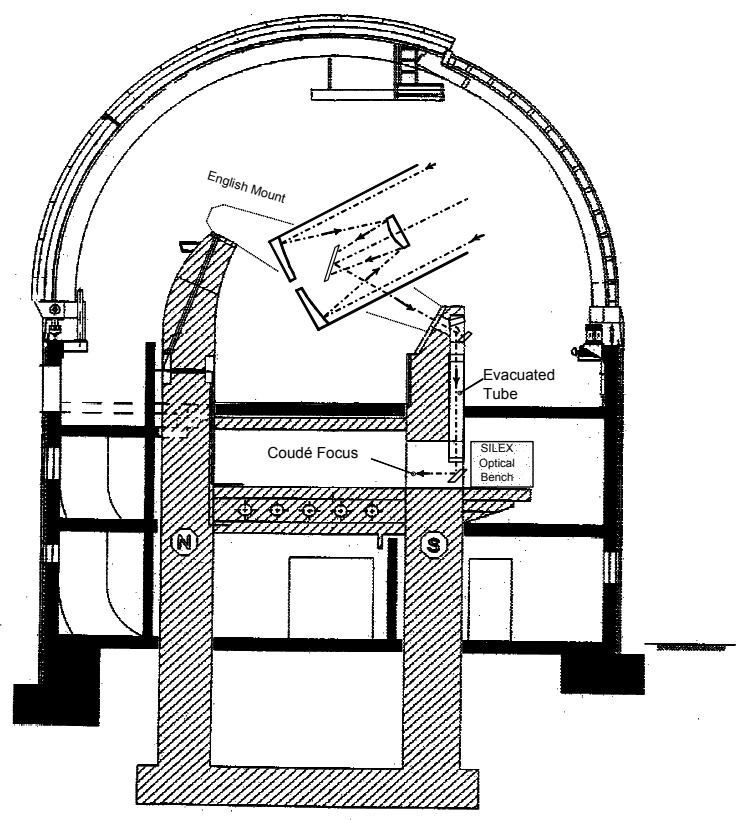

Figure 1: Section through the OGS structure

\section{WAVEFRONT AND INTENSITYMEASUREMENTS AT THE OGS}

Wavefront distortions can be measured with a Shack-Hartmann-Sensor (SHS). An SHS basically consists of a CCDcamera positioned at a pupil of the telescope's beam-path which has an array of small lenses positioned in front of it. Each of these micro-lenses casts its focal spot directly onto the CCD-plane. When the incoming wavefront concludes local tilts the sub-foci at that region deviate laterally. The CCDpictures are transferred to a computer program that detects the center of gravity of each sub focus, compares their positions with a reference-picture and so can calculate the distortions of the incoming wavefront. In addition, post processing of the intensities inside each sub-focus spot allows calculation of the intensity distribution at the aperture. In combination, this processing allows complete description of the phase and amplitude of the incoming wave.

Though the scintillation patterns do depend on the wavelength this impacts receiver performance only slightly (as can be shown by simulation) as the wavefront distortion here is the much more dominant parameter. The SHS used for the wavefront measurements at the OGS had an array of 64x64 microlenses and the CCD's resolution was 1000x1000 pixels, providing about 244 pixels per sub-focus. Measurements were performed in November 1998, in several sessions 
during different times of day. From those measurements a set of 7 sequences was chosen with 10 images each, resulting in 70 single images all in all. The environmental data like elevation, view direction, temperature, wavelength, and wind-velocity and -direction is reported with each sequence (see table 1).

For each sequence a reference wavefront (the one that all single measured wavefronts are compared to) was computed by calculating the average frame of an even larger set of SHS frames.

\begin{tabular}{|c|c|c|l|c|c|c|c|l|}
\hline seq. no. & elevation & time & relative time & wind speed & wind dir. & temp. & view dir. & reference star \\
\hline 1 & $29^{\circ}$ & $06: 45$ & $1 \mathrm{~h}$ before sunrise & $3 \mathrm{~m} / \mathrm{s}$ & $\mathrm{S}$ & $5^{\circ} \mathrm{C}$ & $\mathrm{O}$ & Arcturus \\
\hline 2 & $35^{\circ}$ & $07: 34$ & short before sunrise & $3 \mathrm{~m} / \mathrm{s}$ & $\mathrm{S}$ & $6^{\circ} \mathrm{C}$ & $\mathrm{O}$ & Arcturus \\
\hline 3 & $13^{\circ}$ & $08: 25$ & $1 \mathrm{~h}$ after sunrise & $6 \mathrm{~m} / \mathrm{s}$ & $\mathrm{S}$ & $8^{\circ} \mathrm{C}$ & $\mathrm{W}$ & Beteigeuse \\
\hline 4 & $57^{\circ}$ & $09: 17$ & $2 \mathrm{~h}$ after sunrise & $7 \mathrm{~m} / \mathrm{s}$ & $\mathrm{S}$ & $10^{\circ} \mathrm{C}$ & $\mathrm{O}$ & Arcturus \\
\hline 5 & $62^{\circ}$ & $17: 35$ & $1 \mathrm{~h}$ before sunset & $10 \mathrm{~m} / \mathrm{s}$ & $\mathrm{WNW}$ & $12^{\circ} \mathrm{C}$ & $\mathrm{NW}$ & Vega \\
\hline 6 & $65^{\circ}$ & $17: 57$ & $0.5 \mathrm{~h}$ before sunset & $11 \mathrm{~m} / \mathrm{s}$ & $\mathrm{WNW}$ & $8^{\circ} \mathrm{C}$ & $\mathrm{NW}$ & Vega \\
\hline 7 & $8^{\circ}$ & $20: 05$ & $1.5 \mathrm{~h}$ after sunset & $12 \mathrm{~m} / \mathrm{s}$ & $\mathrm{WNW}$ & $6^{\circ} \mathrm{C}$ & $\mathrm{ONO}$ & Aldebaran \\
\hline
\end{tabular}

Table 1 : Environmental data of the 7 measurement sequences

\section{POST PROCESSING OF THE MEASURED SAMPLES}

\subsection{Field distribution at the aperture}

First the overall tilt underlying each measured sample was removed. This proceeding can be compared with the application of a tip-tilt tracking mirror. The wavefront-samples were transformed to phase-errors by introducing the three widely used laser wavelengths $\lambda_{1}=0.532 \mu \mathrm{m}, \lambda_{2}=1.064 \mu \mathrm{m}$, and $\lambda_{3}=1.550 \mu \mathrm{m}$. The phase errors increase indirect proportional with the wavelength.

In a shot-noise limited receiver the sensitivity is directly connected with the incoming number of photons per bit (e.g. 20 photons per bit for a bit error rate of $10^{-9}$ in a very good homodyne BPSK receiver), thus because photon energy increases proportionally with its frequency, the system working at $0.532 \mu \mathrm{m}$ wavelength will need twice the received power of the one working at $1.064 \mu \mathrm{m}$. On the other hand the transmitter-beam with shorter wavelength has a smaller angle of divergence, at least when propagating in vacuum. When passing through the air, the turbulence-induced beamspreading will again affect the shorter wavelengths stronger than the longer ones, but the evaluation of these effects is not within the scope of this paper. To avoid the evaluation of those further effects along the transmission channel, the energy in each measured sample is equalized here. Thus all samples contain the same amount of energy, no matter which wavelength is being observed. Other wavelength-dependent effects inside the receiver except those influencing the size and shape of the local phase and speckle pattern distribution are being neglected. The normalized fieldamplitude distributions were generated by computing the square-root of the normalized intensity distributions. Figure 3 shows an example of field distribution at the aperture (as measured with the SHS at the pupil). The example-plots in this paper always show the same sample (Seq.1, first sample).

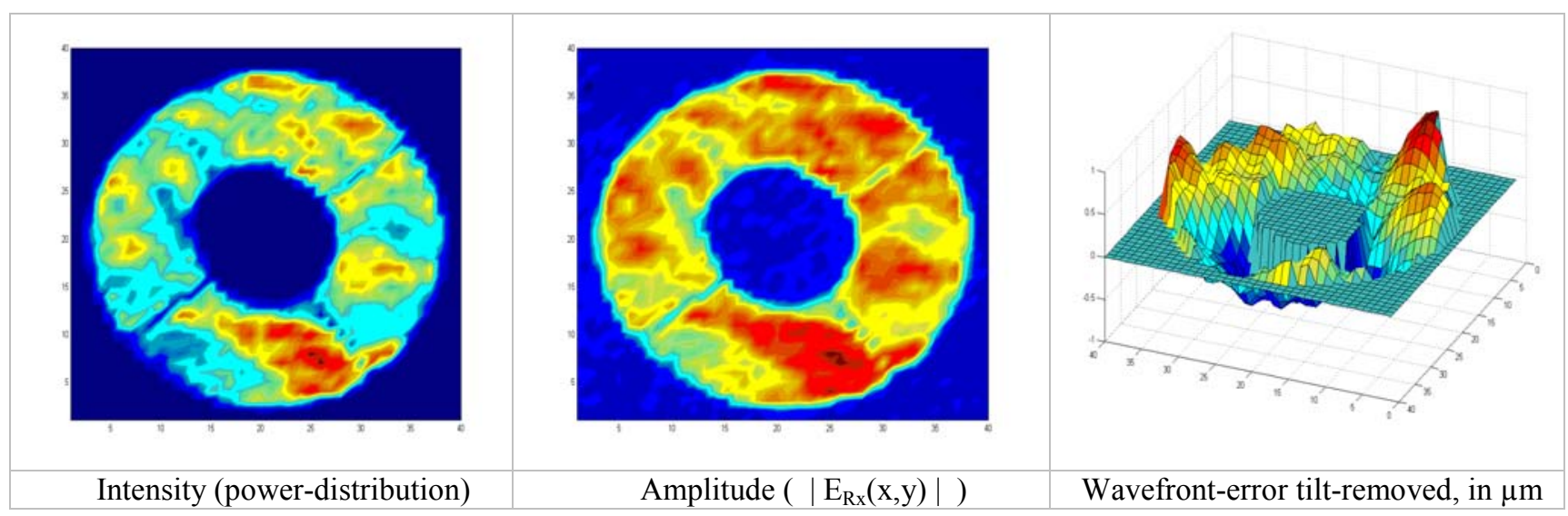

Figure 3 : Sample measurement at the pupil 


\section{2. $r_{0}$-calculations}

The Fried-parameter $r_{0}$ has been defined by D.L. Fried [FRI67] as the minimum aperture size which provides optimum heterodyning with an atmospherically distorted wavefront. Larger apertures than $\mathrm{r}_{0}$ will not improve the efficiency of the receiver significantly. $r_{0}$ is a long-term parameter including overall tilt of the incoming beam over a long exposure time, $r_{0}$ increases with wavelength. By providing tip-tilt-tracking of the wavefront one can improve the telescopes resolution, thus an increased $" \mathrm{r}_{0 \mathrm{tr}}$ " (tilt-removed), regarding all wavefront-samples of one sequence, can be calculated. For a $1 \mathrm{~m}$-telescope (like the OGS) this improvement typically is a factor of 1.5 for $\mathrm{r}_{0}$. When looking at a sequence of samples there will always be some with very small wavefront error, designating an upper limit for the further modified tilt-removed short-term parameter " $r_{0 \text { trst }}$ ". Table 2 summarizes these results for the seven sequences.

The $r_{0}$-values have been calculated by the method described in [GLI97], based on the FWHM-calculation of the focal speckle pattern (focal intensity distributions). The ideal $r_{0 t r s t}$ (in line "ideal" of table 2) are based on the centrally obscured aperture with a uniform intensity and plane wavefront, computed by encircled-energy-in-focal-spot. The longterm $r_{0}$ including tilt have been measured separately, this explains some deviations from the other data.

\begin{tabular}{|c|c|c|c|c|c|c|c|c|c|}
\hline \multirow[t]{2}{*}{ seq. } & \multicolumn{3}{|c|}{$\begin{array}{l}\text { long-term } \mathrm{r}_{0} \text { incl. tilt }\left(\mathrm{r}_{0}\right) \\
\text { in } \mathrm{mm}\end{array}$} & \multicolumn{3}{|c|}{$\begin{array}{l}\text { long-term } \mathrm{r}_{0} \text { tilt-removed }\left(\mathrm{r}_{0 \mathrm{tr}}\right) \\
\text { in } \mathrm{mm}\end{array}$} & \multicolumn{3}{|c|}{$\begin{array}{l}\text { short-term } \mathrm{r}_{0} \text { tilt-removed }\left(\mathrm{r}_{0 \text { trst }}\right) \\
\min / \text { mean } / \max \text { in } \mathrm{mm}\end{array}$} \\
\hline & $0.532 \mu \mathrm{m}$ & $1.064 \mu \mathrm{m}$ & $1.55 \mu \mathrm{m}$ & $0.532 \mu \mathrm{m}$ & $1.064 \mu \mathrm{m}$ & $1.55 \mu \mathrm{m}$ & $0.532 \mu \mathrm{m}$ & $1.064 \mu \mathrm{m}$ & $1.55 \mu \mathrm{m}$ \\
\hline ideal & - & - & - & - & - & - & 553 & 664 & 691 \\
\hline 1 & - & - & - & 64 & 144 & 210 & $54 / 65 / 83$ & $123 / 148 / 195$ & $186 / 222 / 302$ \\
\hline 2 & 51 & 117 & 184 & 66 & 158 & 242 & $57 / 68 / 83$ & $128 / 160 / 195$ & $186 / 248 / 302$ \\
\hline 3 & 37 & 85 & 133 & 61 & 133 & 193 & $40 / 65$ / 98 & 85 / 142 / 221 & $124 / 211 / 302$ \\
\hline 4 & 40 & 92 & 144 & 55 & 123 & 186 & 49 / $56 / 69$ & $104 / 124 / 158$ & $161 / 187 / 230$ \\
\hline 5 & - & - & - & 79 & 174 & 254 & $69 / 81 / 98$ & 144 / 179 / 207 & $220 / 253 / 284$ \\
\hline 6 & 52 & 120 & 188 & 75 & 175 & 269 & $66 / 75 / 92$ & $166 / 180 / 221$ & $242 / 281 / 322$ \\
\hline 7 & 39 & 90 & 141 & 59 & 128 & 193 & $50 / 60 / 87$ & $107 / 132 / 195$ & $161 / 203 / 302$ \\
\hline
\end{tabular}

Table 2: $\mathrm{r}_{0}$-data of the measured samples

\subsection{Focal speckle statistics}

The simulated model has been scaled to the OGS' focal plane in Cassegrain-configuration, which means an effective focal length of $39 \mathrm{~m}$. Together with the $1 \mathrm{~m}$ telescope-aperture with its central obscuration (secondary mirror) of $33 \mathrm{~cm}$ this signifies an airy-like diffraction limited focal spot pattern "Airyco" (centrally obscured) with its first zero-ring at a diameter of $46 \mu \mathrm{m}$ for $\lambda_{1}=0.532 \mu \mathrm{m}\left(92 \mu \mathrm{m} @ \lambda_{2}\right.$, and $137 \mu \mathrm{m} @ \lambda_{3}$ respectively). The observed focal area within the numerical treatment of the samples always is $0.8 \times 0.8 \mathrm{~mm}^{2}$. This practically contains the whole speckle pattern.

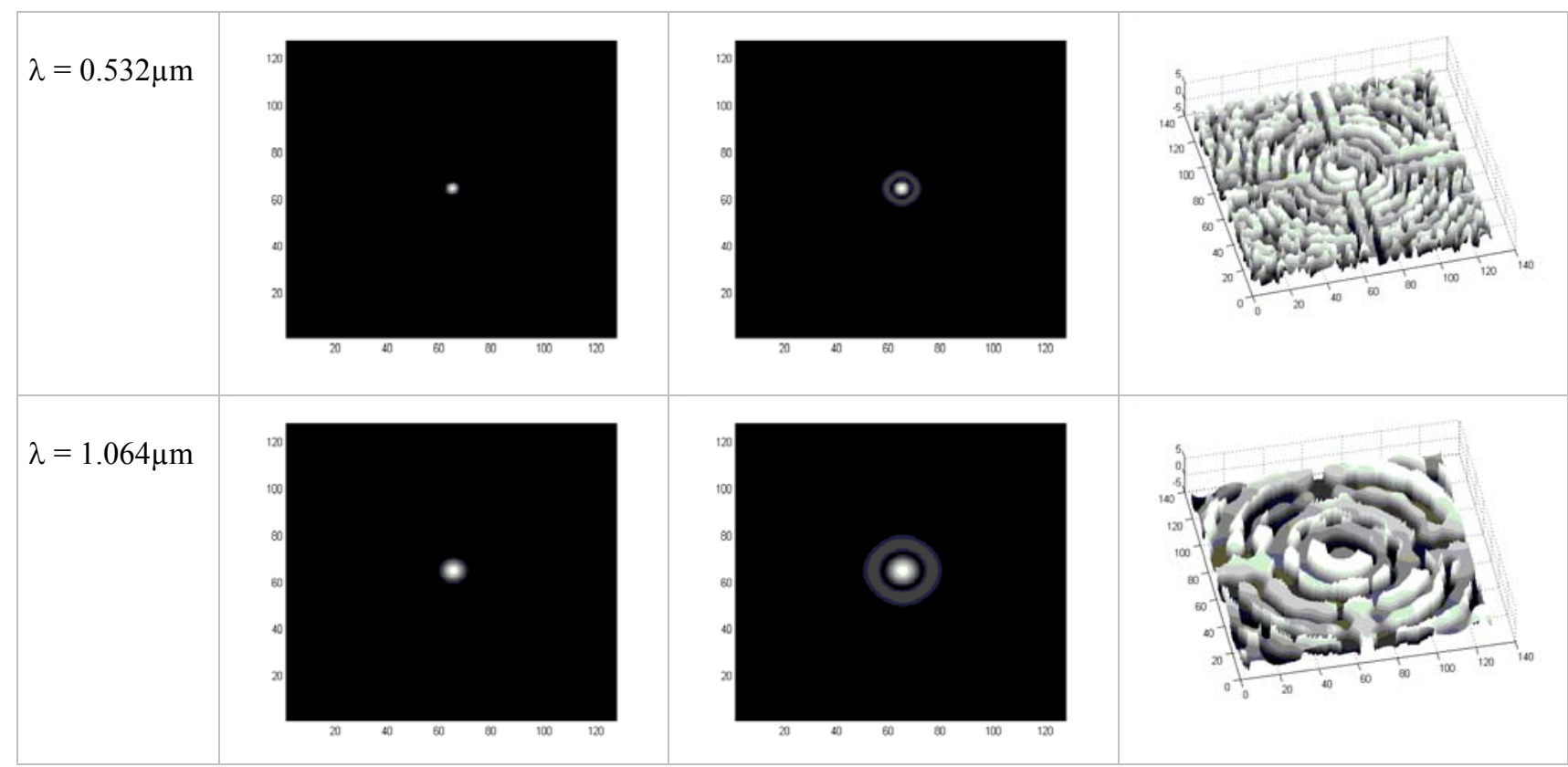




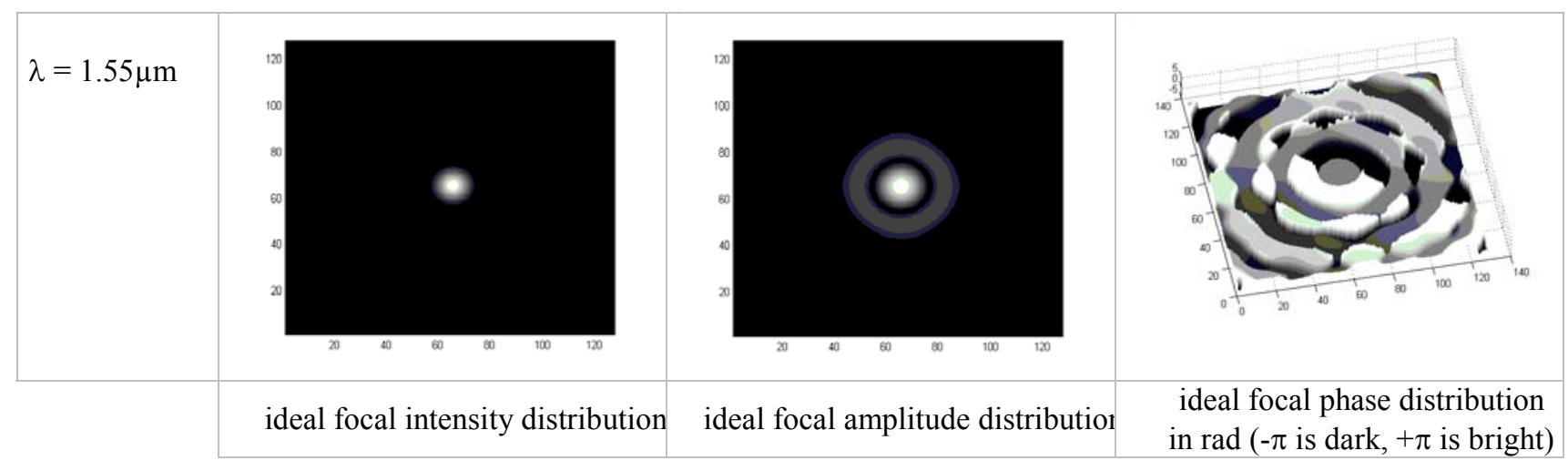

Figure 4 : Ideal focal distributions (plane and equally distributed wavefront onto aperture), square area is $800 \mathrm{x} 800 \mu \mathrm{m}^{2}$

The measured distorted aperture-fields will generate the notorious speckle-patterns at the focal plane. They are calculated by applying a scaled two-dimensional Fourier-Transform onto the aperture-field. As the overall wavefront tilt has been removed from the samples, the center of gravity of all speckle patterns remains on-axis.

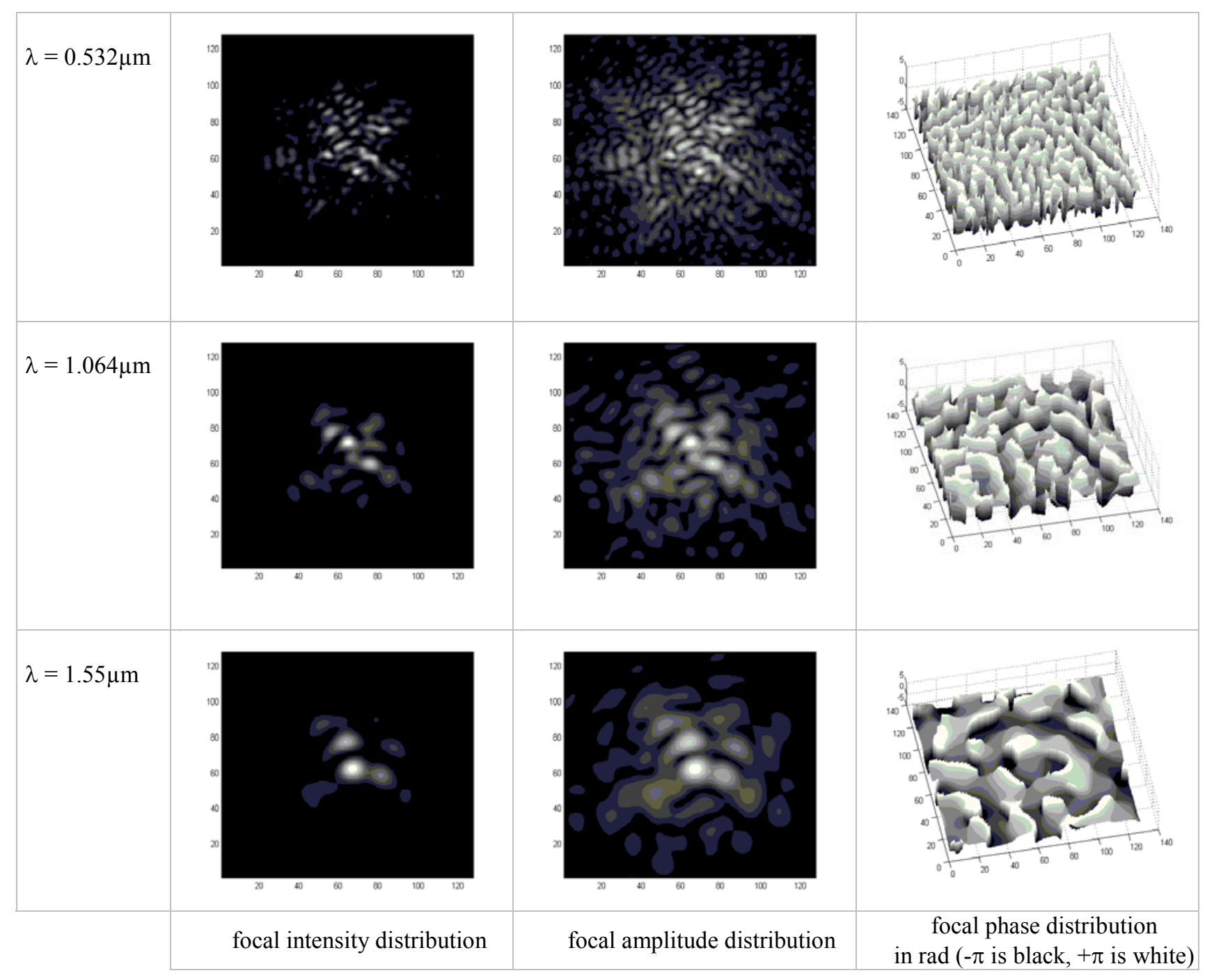

Figure 5 : Sample focal distribution as calculated from aperture-field measurement, square area is $800 \times 800 \mu \mathrm{m}^{2}$ 


\section{SIMULATED FOCAL AND PUPIL HETERODYNING EFFICIENCIES}

\subsection{System setup}

The simulated coherent receiver setup is shown in figure 6 . To use the measurements from the referencestars the following assumptions have been made:

- the spacecraft with the optical transmitter is far enough away to treat the wave above the atmosphere as plane and equally distributed (i.e. at least MEO-satellite)

- the tip-tilt tracking mechanism is capable of perfect center-of-gravity-tracking of the focal speckle pattern

Figure 7 explains the processing of the wavefront samples. Superpositioning with the local oscillator (LO) can also take place at the pupil, thereby avoiding the fourier-transform. In a real receiver setup this is usually not done because of the large detector area necessary, which limits the system's bandwidth. [FRI67] explains that with an untracked beam (no tiptilt tracking) the maximum achievable heterodyning efficiencies will be the same for focal as well as for pupil heterodyning. However with tip-tilt-tracking focal heterodyning shows a slight improvement (see tables 3 and 4). In section 5.3 a special pupilsuperpositioning technique is explained.

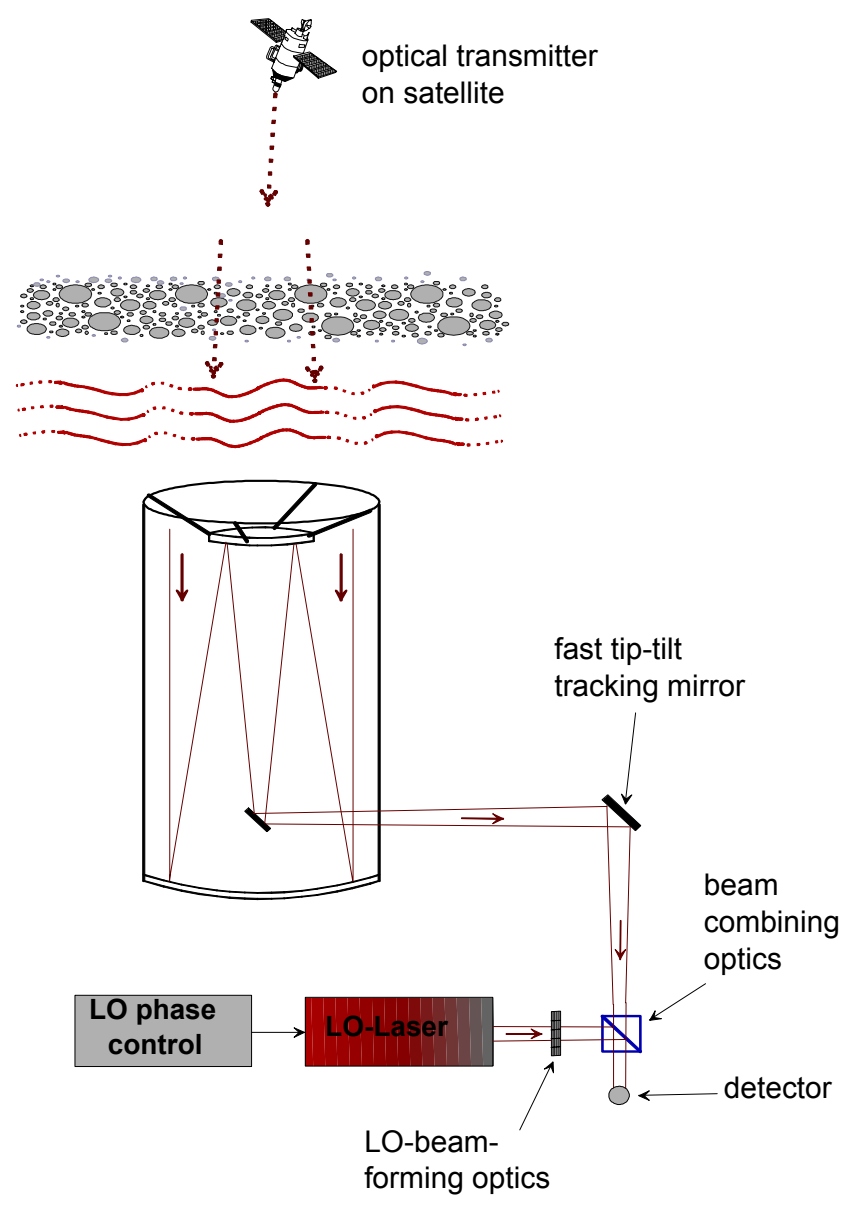

Figure 6 : Setup as represented by the heterodyning-efficiencysimulation

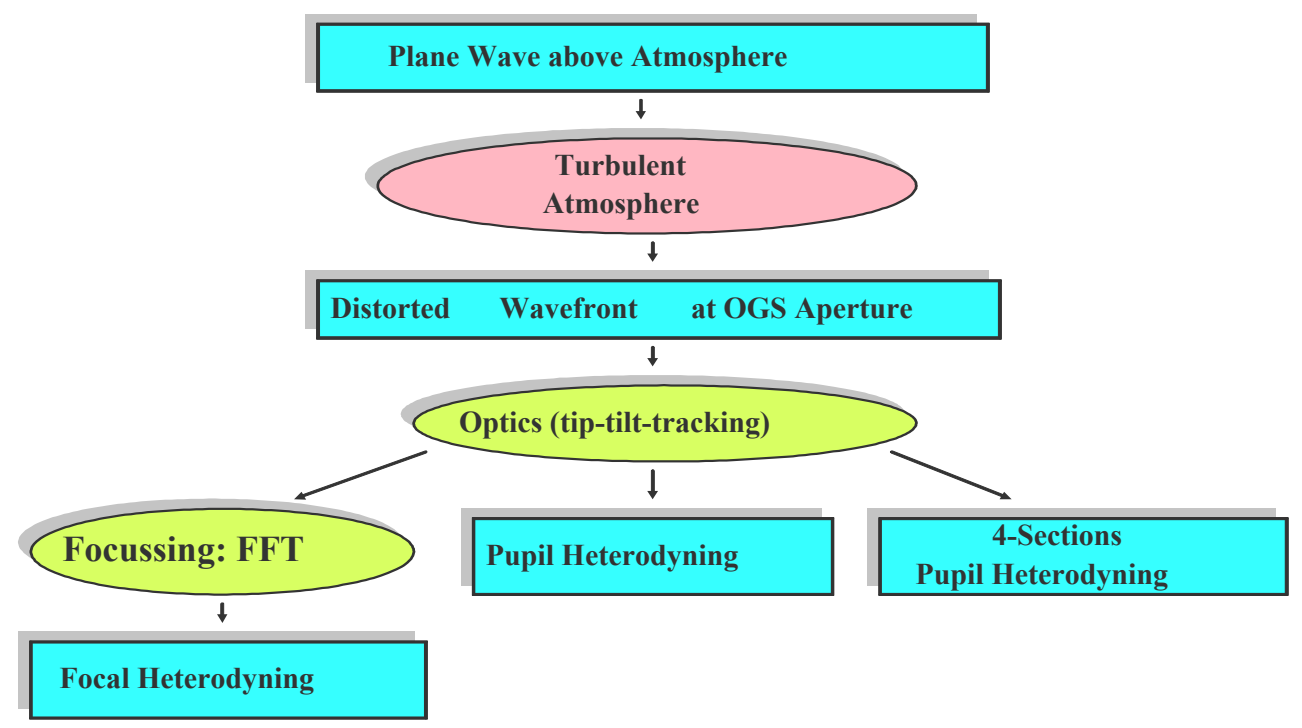

Figure 7 : Processing-diagram of the simulated system 


\subsection{Heterodyning efficiency with complex wavefronts and speckles}

By computing the Heterodyning Efficiency $\eta_{H E}$ for the electrical signal power the match between the shapes of the Rxand LO-field is considered. With non-complex fields (undistorted wavefronts) this can be calculated by applying [COH75]:

$$
\eta_{H E}=\frac{\left|\iint_{P D} E_{L O} \cdot E_{R x} d x d y\right|^{2}}{\iiint_{P D}\left|E_{L O}\right|^{2} d x d y \cdot \iint\left|E_{R x}\right|^{2} d x d y}
$$

with $\mathrm{E}_{\mathrm{LO}}$ and $\mathrm{E}_{\mathrm{Rx}}$ denoting the electric fields of the local oscillator and the received light respectively and PD the area of the photo-detector.

A distorted wavefront is represented by a complex field distribution and thus the $\eta_{\mathrm{HE}}$-formula has to be extended [CHA97]:

$$
\eta_{H E}=\frac{\iint_{P D} E_{L O} \cdot E_{R x}^{*} d x d y \cdot \iint_{P D} E_{L O}{ }^{*} \cdot E_{R x} d x d y}{\iiint_{P D}\left|E_{L O}\right|^{2} d x d y \cdot \iint\left|E_{R x}\right|^{2} d x d y}
$$

In the case of homodyne phase shift keying reception the LO-phase has to be matched with the speckle pattern to provide optimum SNR. This will result in a factor 2 increase compared to heterodyne reception but with the wrong LOphase the efficiency will drop to zero. In this paper no further attention will be paid to the type of receiver or modulation thus regarding only the general $\eta_{\mathrm{HE}}$ is sufficient for comparison.

The computing of efficiencies requires a certain integration-area representing the photo-detector (PD) size and shape. In our model the detector has a uniform responsivity over its whole area and a shape matching that of the non-zero part of the LO-field. I.e. in the case of pupil superpositioning (table 4) detector and LO have the shape of the segmented

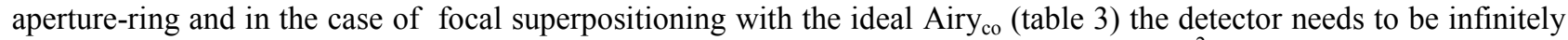
large. However, the numerically regarded focal area is always limited to $800 \mathrm{x} 800 \mu \mathrm{m}^{2}$ in the simulation which is

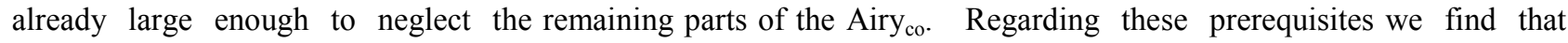
$\mathrm{SNR} \sim \eta_{\mathrm{HE}}$.

By comparing the results for the different wavelengths with each other one has to remember that receiver sensitivity increases proportionally with wavelength because sensitivity is proportional to the number of received photons. The mean SNR-degradation in tables 3 and 4 regards this fact and thus compares the $1.064 \mu \mathrm{m}$ - and $0.532 \mu \mathrm{m}$-systems with the one working at $1.55 \mu \mathrm{m}$ when constant power onto the aperture is guaranteed (free-space beam-spreading losses have to be regarded separately).

\begin{tabular}{|r|c|c|c|c|c|c|}
\hline \multirow{2}{*}{ seq. } & \multicolumn{3}{|c|}{$\begin{array}{c}\text { focal heterodyning efficiencies } \\
\text { min / mean / max }\end{array}$} & \multicolumn{3}{c|}{$\begin{array}{c}\text { mean SNR-degradation with } \\
\text { focal het. \& equal power in dB }\end{array}$} \\
\cline { 2 - 7 } & $\mathbf{0 . 5 3 2 \mu m}$ & $\mathbf{1 . 0 6 4 \mu \mathbf { m }}$ & $\mathbf{1 . 5 5} \boldsymbol{\mu m}$ & $\mathbf{0 . 5 3 2 \mu m}$ & $\mathbf{1 . 0 6 4} \boldsymbol{\mu m}$ & $\mathbf{1 . 5 5} \boldsymbol{\mu m}$ \\
\hline ideal & 1 & 1 & 1 & -4.65 & -1.64 & 0 \\
\hline 1 & $0.0012 / 0.00783 / 0.027$ & $0.0020 / 0.0258 / 0.071$ & $0.0038 / 0.13 / 0.317$ & -25.7 & -17.4 & -8.9 \\
\hline 2 & $0.0005 / 0.0118 / 0.017$ & $0.017 / 0.0935 / 0.19$ & $0.045 / 0.285 / 0.42$ & -23.9 & -11.9 & -5.5 \\
\hline 3 & $0.000055 / 0.0081 / 0.053$ & $0.00086 / 0.0375 / 0.11$ & $0.0040 / 0.12 / 0.34$ & -25.5 & -15.9 & -9.2 \\
\hline 4 & $0.00058 / 0.0067 / 0.035$ & $0.014 / 0.0402 / 0.094$ & $0.0052 / 0.13 / 0.30$ & -26.4 & -15.5 & -8.8 \\
\hline 5 & $0.00018 / 0.0093 / 0.033$ & $0.0061 / 0.077 / 0.24$ & $0.052 / 0.22 / 0.55$ & -24.9 & -12.8 & -6.6 \\
\hline 6 & $0.00018 / 0.010 / 0.033$ & $0.01 / 0.078 / 0.17$ & $0.0048 / 0.24 / 0.43$ & -24.7 & -12.7 & -6.2 \\
\hline 7 & $0.00018 / 0.0068 / 0.024$ & $0.0051 / 0.036 / 0.13$ & $0.012 / 0.085 / 0.37$ & -26.4 & -16.0 & -10.7 \\
\hline
\end{tabular}

Table 3: Focal receiver efficiency statistics 


\begin{tabular}{|l|c|c|c|c|c|c|}
\hline \multirow{2}{*}{ seq. } & \multicolumn{3}{|c|}{$\begin{array}{c}\text { pupil heterodyning efficiencies } \\
\text { min } / \text { mean } / \text { max }\end{array}$} & \multicolumn{2}{c|}{$\begin{array}{c}\text { mean SNR-degradation with } \\
\text { pupil het. \& equal power in dB }\end{array}$} \\
\cline { 2 - 7 } & $\mathbf{0 . 5 3 2 \mu \mathrm { m }}$ & $\mathbf{1 . 0 6 4} \boldsymbol{\mu m}$ & $\mathbf{1 . 5 5} \boldsymbol{\mu m}$ & $\mathbf{0 . 5 3 2} \boldsymbol{\mu m}$ & $\mathbf{1 . 0 6 4} \boldsymbol{\mu m}$ & $\mathbf{1 . 5 5} \boldsymbol{\mu m}$ \\
\hline ideal & 1 & 1 & 1 & -4.65 & -1.64 & 0 \\
\hline 1 & $0.0012 / 0.00786 / 0.027$ & $0.0015 / 0.023 / 0.066$ & $0.0025 / 0.115 / 0.287$ & -25.7 & -18.0 & -9.2 \\
\hline 2 & $0.0005 / 0.0053 / 0.017$ & $0.017 / 0.0846 / 0.17$ & $0.04 / 0.213 / 0.36$ & -27.4 & -12.4 & -6.8 \\
\hline 3 & $0.000044 / 0.0029 / 0.012$ & $0.00072 / 0.0302 / 0.11$ & $0.0032 / 0.11 / 0.31$ & -30.0 & -16.8 & -9.6 \\
\hline 4 & $0.00053 / 0.0067 / 0.035$ & $0.012 / 0.0354 / 0.088$ & $0.0036 / 0.11 / 0.27$ & -26.4 & -16.2 & -9.6 \\
\hline 5 & $0.0002 / 0.011 / 0.033$ & $0.0015 / 0.070 / 0.22$ & $0.043 / 0.19 / 0.49$ & -24.2 & -13.2 & -7.2 \\
\hline 6 & $0.00020 / 0.012 / 0.018$ & $0.0095 / 0.070 / 0.15$ & $0.042 / 0.21 / 0.39$ & -23.9 & -13.2 & -6.8 \\
\hline 7 & $0.00016 / 0.0068 / 0.024$ & $0.00082 / 0.0307 / 0.11$ & $0.019 / 0.071 / 0.32$ & -26.4 & -16.8 & -11.5 \\
\hline
\end{tabular}

Table 4: Pupil receiver efficiency statistics

\subsection{Section-aperture receiver / multi-aperture approach:}

One suggestion to cope with the wavefront distortion is to split up the large $1 \mathrm{~m}$ aperture into several subapertures, which will lead to smaller phase errors and thus enhance heterodyne efficiency in each subaperture. For a heterodyne receiver the single electrical signals then must be phase-corrected before summing them up into one. In the case of homodyne reception the LO-phases for each segment have to be controlled for optimum $\eta_{\mathrm{HE}}$ before superpositioning, which leads to some additional expense in hardware. These kinds of receivers have been suggested by [GOL65] and described by [GAT]. According to [FRI67], by reducing the aperture down to the size of the Fried-parameter " $\mathrm{r}_{0}$ " the optimum HE will occur. As shown in table 2 the $\mathrm{r}_{0}$ of the $1.55 \mu \mathrm{m}$ samples with tilt-removed is around $250 \mathrm{~mm}$, which comes close to the investigated sub-aperture size. Here we will concentrate on the special case of four separate ringsegments in the pupil plane (matching the four segments of the Cassegrain-aperture), each heterodyned with its optimum LO-phase and -shape and then recombined electrically. With this proceeding the aperture-sums and thus the amount of collected power remain the same as in the previous setup. The overall HE is the mean of all four sub-HEs.

\begin{tabular}{|c|c|c|c|c|c|c|}
\hline \multirow[t]{2}{*}{ seq. } & \multicolumn{3}{|c|}{$\begin{array}{l}\text { 4-section pupil heterodyning efficiency } \\
\text { min / mean / max }\end{array}$} & \multicolumn{3}{|c|}{$\begin{array}{l}\text { mean SNR-degradation with 4-section } \\
\text { pupil heterodyning \& equal power in } \mathrm{dB}\end{array}$} \\
\hline & $0.532 \mu \mathrm{m}$ & $1.064 \mu \mathrm{m}$ & $1.55 \mu \mathrm{m}$ & $0.532 \mu \mathrm{m}$ & $1.064 \mu \mathrm{m}$ & $1.55 \mu \mathrm{m}$ \\
\hline ideal & 1 & 1 & 1 & -4.65 & -1.64 & 0 \\
\hline 1 & $0.014 / 0.027 / 0.037$ & $0.030 / 0.11 / 0.187$ & $0.172 / 0.27 / 0.41$ & -20.3 & -11.2 & -5.7 \\
\hline 2 & $0.0093 / 0.025 / 0.045$ & $0.077 / 0.17 / 0.26$ & $0.17 / 0.34 / 0.47$ & -20.7 & -9.2 & -4.7 \\
\hline 3 & $0.0022 / 0.036 / 0.12$ & $0.012 / 0.14 / 0.46$ & $0.025 / 0.26 / 0.65$ & -19.2 & -10.2 & -5.9 \\
\hline 4 & $0.0071 / 0.021 / 0.066$ & $0.056 / 0.092 / 0.14$ & $0.126 / 0.23 / 0.32$ & -21.4 & -12 & -6.4 \\
\hline 5 & $0.012 / 0.046 / 0.089$ & $0.071 / 0.19 / 0.32$ & $0.19 / 0.36 / 0.56$ & -20.0 & -8.9 & -4.4 \\
\hline 6 & $0.014 / 0.037 / 0.057$ & $0.14 / 0.20 / 0.31$ & $0.31 / 0.40 / 0.54$ & -18.9 & -8.5 & -4.0 \\
\hline 7 & $0.0072 / 0.018 / 0.051$ & $0.047 / 0.087 / 0.20$ & $0.11 / 0.20 / 0.36$ & -22.1 & -12.2 & -7.0 \\
\hline
\end{tabular}

Table 5: Efficiency of 4-section pupil receiver 


\section{RESULTS AND SUMMARY}

The results of the heterodyne efficiency simulation are summarized in Figures 8 to 13. A tendentious though not exactly functional contiguity between $r_{0}$ and $\eta_{\mathrm{HE}}$ can be observed in figures 8 and 10. The dotted fit-curve in figure 8 is $0.25^{*}\left(\mathrm{r}_{0} / 300 \mathrm{~mm}\right)^{2}$ and in figure 10 it is $0.5^{*}\left(\mathrm{r}_{0} / 300 \mathrm{~mm}\right)^{2} \quad\left(\mathrm{r}_{0}\right.$ here always is short-term tilt-removed). In figures 9 and 11 the dependence of $\eta_{\mathrm{HE}}$ on the wavelength can be seen. The numbers at the plots refer to the sequences and the dotted fit-curve in figure 9 is $0.055 * \lambda^{3}$ and $0.12 * \lambda^{2}$ in figure 11 . Again it has to be remarked that these figures are for equal received power and thus do not represent the overall performance of the optical communication link.

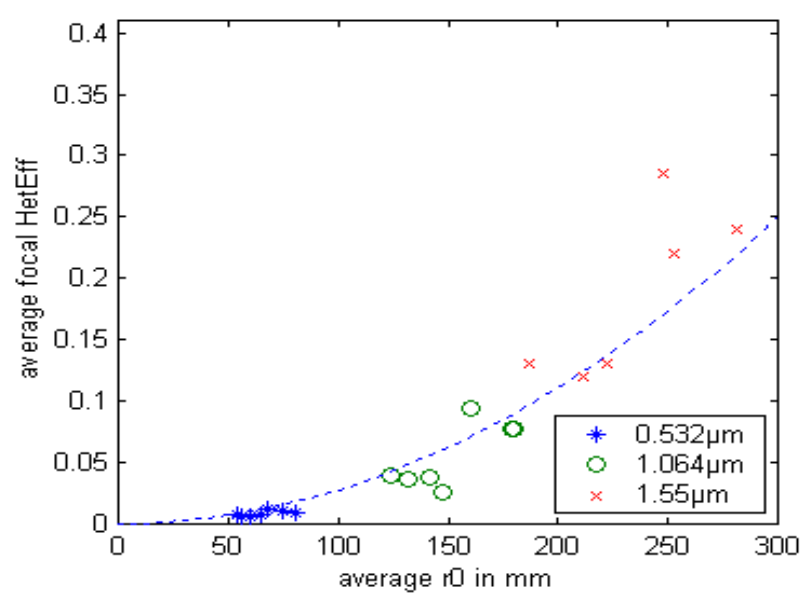

Figure 8: Focal $\eta_{\text {HE }}$ over $r_{0}$

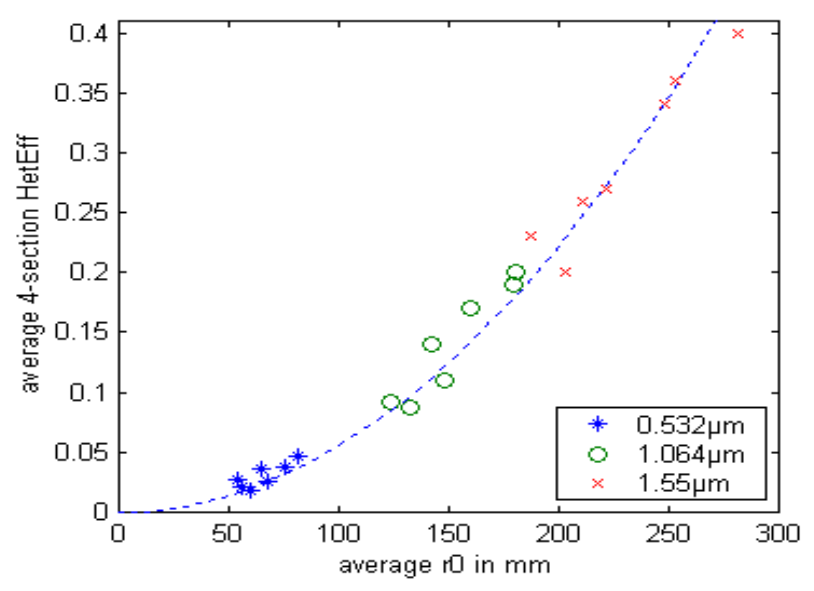

Figure 10: 4-sections $\eta_{\mathrm{HE}}$ over $\mathrm{r}_{0}$

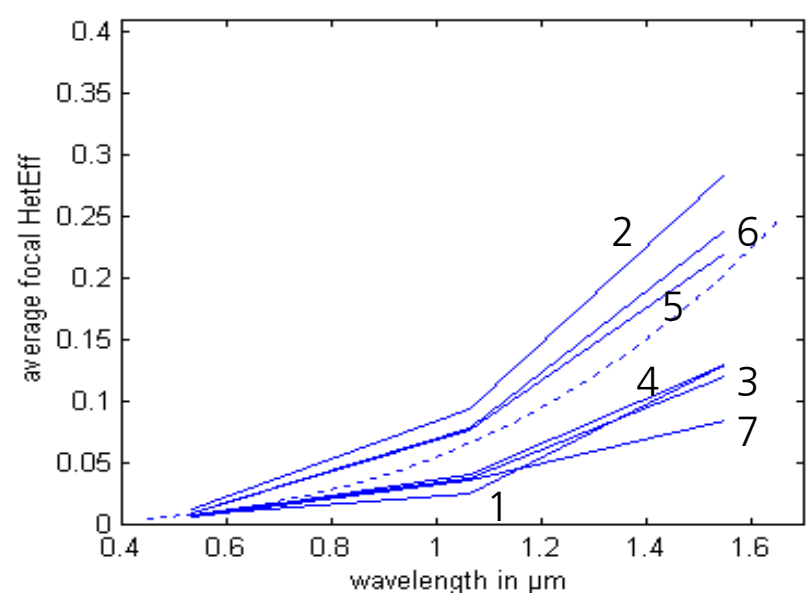

Figure 9: Focal $\eta_{\text {HE }}$ over $\lambda$

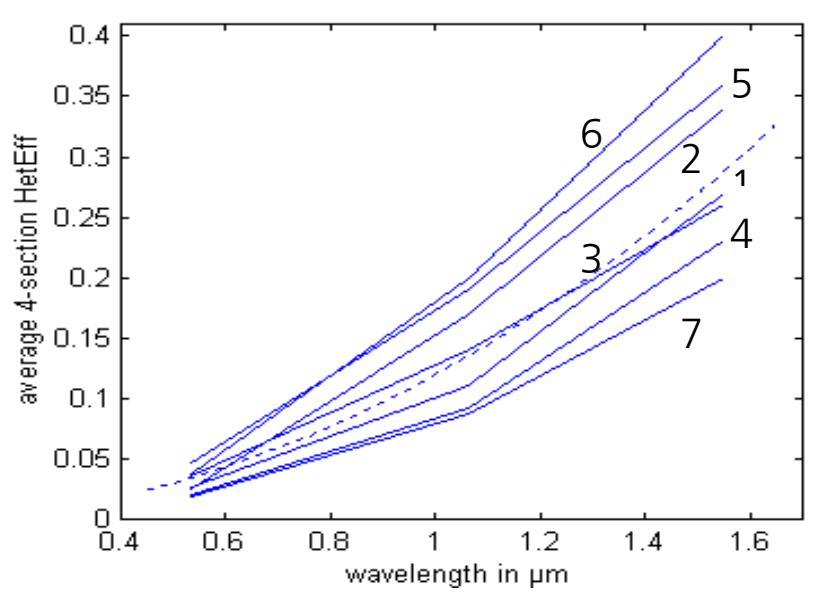

Figure 11: 4-sections $\eta_{\mathrm{HE}}$ over $\lambda$

It is obvious that $\eta_{\mathrm{HE}}$ increases with wavelength, bringing communication links working with $1.55 \mu \mathrm{m}$ into a useful range with an all-sequences-average- $\eta_{\mathrm{HE}}$ of $17 \%$ for the simple focal heterodyning receiver and $21 \%$ with the 4 -section receiver. But this has to be traded off with the overall communications system e.g. with the received power: the divergence angle of the optical transmit beam grows inverse proportional with wavelength, leading to a lower power concentration at the receiver side. Furthermore, coherent systems with laser diodes $(1.55 \mu \mathrm{m})$ only work with high data rates due to the instability in frequency. This makes laser-diodes unemployable for low-rate deep-space links. Here highly stable solid state lasers (e.g. Nd-YAG at $1.064 \mu \mathrm{m})$ are necessary. This wavelength $(1.064 \mu \mathrm{m})$ results in an allsequences-average- $\eta_{\mathrm{HE}}$ of $5.5 \%$ for the focal receiver and $11 \%$ for the 4 -section receiver. Obviously $532 \mathrm{~nm}$ is out of the question from being used for coherent reception under atmospheric turbulence conditions. 


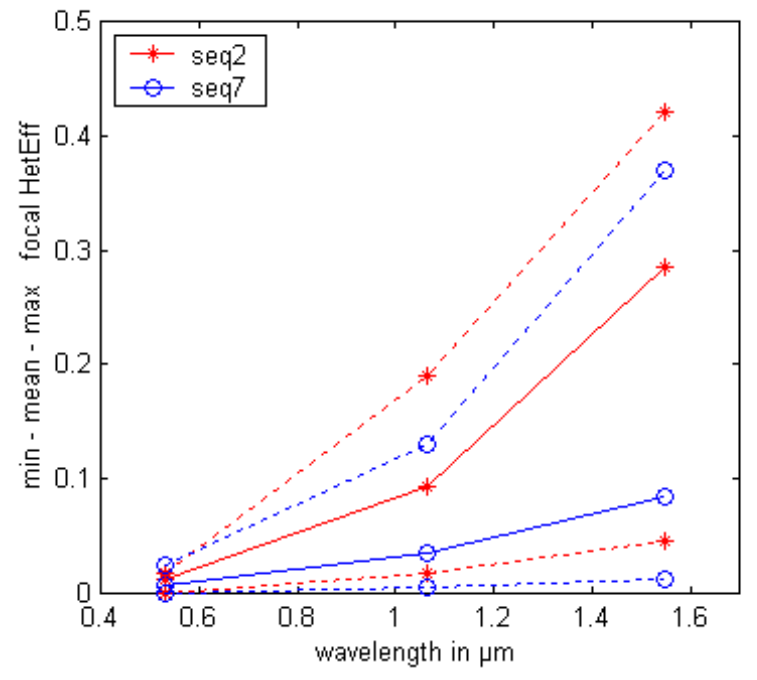

Figure 12: Min-mean-max of focal $\eta_{\mathrm{HE}}$ over $\lambda$

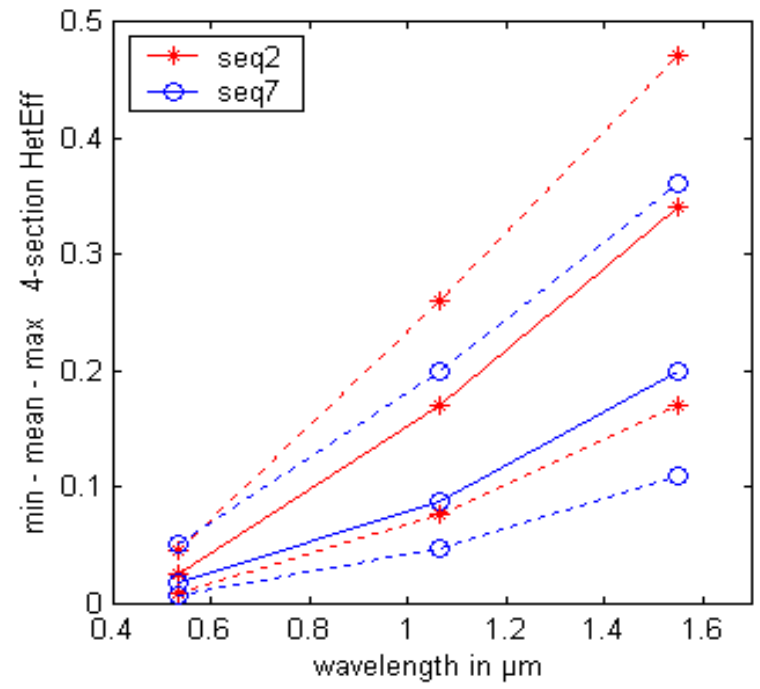

Figure 13: Min-mean-max of 4-sections $\eta_{\mathrm{HE}}$ over $\lambda$

The great advantage of the 4-section system is not only the increased average $\eta_{\mathrm{HE}}$ but even more the increased minimum values (about ten times increase for bad situations like seq. 7 and about four times for good situations such as in seq. 2 as can be seen in figures 12 and 13). These minimum efficiency values are crucial in a communications link as they denote the system sensitivity without encountering burst errors. A practical system has to be designed around these minimum values to be considered as reliable for a given data rate.

The variations in wavefront error are strong under all environmental conditions, thus low heterodyning efficiencies have to be expected in any case and no save lower limit can be given. The probability for higher $\eta_{\mathrm{HE}}$ increases with higher elevations (seq. 5 and 6) and at night-time (seq. 2), while seq. 7 demonstrates the negative impact of the large air mass at low elevations. This speaks in favor of the use of channel-coding techniques that adapt coding-overhead to the environmental conditions. To provide a reliable and efficient receiver certainly higher order multi-aperture systems or adaptive optics or other techniques have to be employed.

\section{ACKNOWLEDGMENTS}

The author whishes to thank Dr. Zoran Sodnik of ESTEC for his help in operating the OGS-Telescope during the measurement sessions even at the most abnormal times of day. The SHS-hardware was kindly provided by Dr. Weidlich of Carl Zeiss, Oberkochen, Germany. The measurement campaign took place within the scope of the "Study for the Adaption of ESA's OGS for Coherent Communication at 1064nm" [OGS99], which has been resourced by DLR's Project Office for Telecommunications and Navigation, Bonn, Germany.

\section{ACRONYMS AND TERMS}

SHS Shack-Hartmann Sensor

PD photo-detector

pupil position in the telescope's optical path where a clear picture of the aperture is formed

focus position of beam-convergence in a telescope's optical path where the optical field forms the 2D-FFT of the aperture's field distribution

$\mathrm{E}_{\mathrm{Rx}} \quad$ field of received light

$\mathrm{E}_{\mathrm{LO}} \quad$ field of local oscillator light

$\eta_{\mathrm{HE}} \quad$ Heterodyning Efficiency factor

SNR signal-to-noise-ratio

FWHM Full Width Half Maximum

Airy $_{\text {co }}$ Airy-like focal diffraction pattern, as it is produced by the centrally-obscured aperture of a Cassegrain-telescope

MEO Medium Earth Orbit (satellite-orbit, around 1500km to $20000 \mathrm{~km}$ above earth) 


\section{REFERENCES}

[CHA97] D.M. Chambers, "Modeling heterodyne efficiency for coherent laser radar in the presence of aberrations", OPTICS EXPRESS, OSA, Vol. 1, No. 3 , August 4, 1997, Page 60

[COH75] S.C. Cohen, "Heterodyne detection: phase front alignment, beam spot size, and detector uniformity", Applied Optics, Vol. 14 , No.8, August 1975

[FRI67] D.L. Fried, "Optical Heterodyne Detection of an Atmospherically Distorted Signal Wave Front", Proceedings of the IEEE, Vol.55, No.1, January 1967

[GAT] P. Gatt, T. Costello, D. Heimmermann, D. Castellanos, A.R. Weeks, C.M. Stickley, "Coherent Optical Array Receivers for the Mitigation of Atmospheric Turbulence and Speckle Effects", submitted to Applied Optics

[GLI97] A. Glindemann, "Beating the Seeing Limit - Adaptive Optics on Large Telescopes", Habilitationsschrift, Rupprecht-Karls-Universität, Heidelberg, 1997

[GOL65] I. Goldstein, P.A. Miles, A. Chabot, "Heterodyne measurements of light propagation through atmospheric turbulence", Proceedings of the IEEE 53, 1172-1180, 1965

[OGS99] "Adaption der OGS für Space to Ground Experimente mit kohärenter 1064nm YAG-LaserKommunikationstechnologie" , concept study by Carl Zeiss, Oberkochen, and DLR-Oberpfaffenhofen, financed by DLR-Bonn, June 1999

[SOD] Zoran Sodnik, Reinhard Czichy, "Design Data Summary of the ESA Optical Ground Station for In-Orbit Check-Out of Laser Communication Payloads and for the Observation and Registration of Space Debris", ESA/ESTEC, Instrument Technology Division 
Institut für

Kommunikation und Navigation

Das Institut für Kommunikation und Navigation betreibt im Vorfeld industrieller Entwicklungen langfristig angelegte ingenieurwissenschaftliche Forschung auf den Gebieten Kommunikation, Navigation und Leitsysteme für Anwendungen in Luftfahrt, Raumfahrt und Verkehr. Hierbei bearbeitet das Institut die Geschäftsfelder Satellitenkommunikation, aeronautische Kommunikation, terrestrische Funksysteme, Satellitennavigation und Verkehrsleitsysteme. 